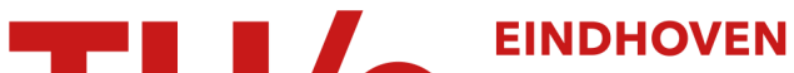 \\ UNIVERSITY OF \\ TECHNOLOGY
}

\section{Multiple-scattering theory beyond the Muffin-Tin approximation}

Citation for published version (APA):

Molenaar, J. (1987). Multiple-scattering theory beyond the Muffin-Tin approximation. (WD report; Vol. 8705).

Radboud Universiteit Nijmegen.

Document status and date:

Published: 01/01/1987

\section{Document Version:}

Publisher's PDF, also known as Version of Record (includes final page, issue and volume numbers)

\section{Please check the document version of this publication:}

- A submitted manuscript is the version of the article upon submission and before peer-review. There can be important differences between the submitted version and the official published version of record. People interested in the research are advised to contact the author for the final version of the publication, or visit the $\mathrm{DOI}$ to the publisher's website.

- The final author version and the galley proof are versions of the publication after peer review.

- The final published version features the final layout of the paper including the volume, issue and page numbers.

Link to publication

\section{General rights}

Copyright and moral rights for the publications made accessible in the public portal are retained by the authors and/or other copyright owners and it is a condition of accessing publications that users recognise and abide by the legal requirements associated with these rights.

- Users may download and print one copy of any publication from the public portal for the purpose of private study or research.

- You may not further distribute the material or use it for any profit-making activity or commercial gain

- You may freely distribute the URL identifying the publication in the public portal.

If the publication is distributed under the terms of Article 25fa of the Dutch Copyright Act, indicated by the "Taverne" license above, please follow below link for the End User Agreement:

www.tue.nl/taverne

Take down policy

If you believe that this document breaches copyright please contact us at:

openaccess@tue.nl

providing details and we will investigate your claim. 
Report WD $87-05$

Multiple-Scattering Theory Beyond

The Muffin-Tin Approximation

J. Molenaar

June 1987

Wiskundige Dienstverlening Faculteit der Wiskunde en Natuurwetenschappen Katholieke Universiteit Toernooiveld 6525 ED Nijmegen The Netherlands 


\title{
Multiple-Scattering Theory Beyond The \\ Muffin-Tin Approximation
}

\author{
J. Molenaar \\ Mathematics Consulting Department \\ Katholieke Universiteit \\ Toernooiveld \\ 6525 ED Nijmegen
}

\begin{abstract}
We present a new derivation of multiple-scattering theory as applied in solid state physics. The present approach is a generalization of the well-known Korringa, Kohn and Rostoker (KKR) formalism and holds also in the case of overlapping potentials. The resulting equations have the same structure as the KKR equations, except for extra energy integrations. It is shown that for non-overlapping potentials these integrations can be performed analytically. Then, they reduce to the KKR equations, in which all matrices are evaluated at one and the same energy . The introduction of a Muffin-Tin potential appears to be not essential in this reduction. From this it is concluded that the so-called "Near Field" corrections, introduced in previous papers dealing with non-Muffin-Tin potentials, can be ignored.
\end{abstract}

\section{\$1. Introduction}

Since the innovative work of Williams and Van Morgan in 1972 and 1974 many authors have tried to extend multiple scattering theory for solids, as developed by Korringa (1947) and Kohn and Rostoker (KKR) (1954), beyond the Muffin-Tin (MT) approximation. In this approximation all potentials are confined to non-overlapping spheres. This geometrical restriction is closely connected to the use of the angular momentum representation in the KKR formalism. Thanks to the finite range of the scattering potentials, the $t$-matrix elements in this representation are in most applications negligible as soon as $l>2$ or 3. The KKR matrix, from which the electronic band structure is obtained, has consequently the same reduced dimensions. This feature, together with a nice separation between lattice and potential dependent quantities, is the reason why the KKR approach has become so extremely popular and fruitful. However, although the MT approximation yields good results in densely-packed structures, it seriously obstructs wider applications. It is therefore not surprising that this approximation has been subject of intense discussions. These concentrate on the (possible) existence and form of so-called Near-Field (NF) corrections, which would strongly diminish the attractiveness of the theory. Because the attempts to cir- 
cumvent the MT approximation have been quite diverse, a lot of confusion has been raised about the validity of the different methods. In the discussion in section 4 we shortly deal with the several approaches in comparison with the present work, pointing out that they rather complement than contradict each other. The present state of the discussion on NF corrections is still appropriately characterized by Faulkner (1979), who stated that "the problem of carrying out a rigorous multiple-scattering calculation for potentials that cannot be put in the form of anisotropic Muffin-Tin potentials is more difficult than it appeared to be in previous discussions" . A tentative conclusion from the different contributions might be that the NF corrections, although they may exist in theory, are small and even negligible in practice.

In this paper we present an extension of the KKR formalism. We show that it is possible to derive equations with the well-known KKR structure, but with a wider applicability. The results are, for example, independent of the used representation and allow the potentials to overlap. The present approach contains in the first instance energy integrations. That is why we like to refer to it as an off-the-energyshell analysis. In the literature only on-the-shell derivations are known. The off-the-shell technique has extensively been dealt with earlier by the present author (1983) but only in the context of the MT approximation. Here we shall release this restriction, meanwhile showing that the NF corrections do not exist.

In section 2 we present the theory in its most general form. Although the resulting formulae might be useless from a practical point of view, they form the necessary starting point for further work. In section 3 we introduce the assumption of non-overlapping potentials and specify the representation to be the energy-angular momentum representation. Then we show that the general expressions in section 2 reduce to the usual KKR equations. The essential tool for this reduction is a theorem, presented in appendix $\mathrm{B}$, by which the energy integrations can be performed analytically. In section 4 we relate the present work to that of other authors.

Throughout this paper atomic units are used with $\hbar=2 m=1, h$ and $m$ being Planck's constant and the electron mass respectively.

\section{\$2. Multiple-Scattering Theory for Overlapping Potentials}

\subsection{Free Space}

We start our considerations with the description of a particle in free space. This system will serve as reference system in the Lipmann-Schwinger equations to be used later on. Denoting the hamiltonian and momentum operator by $H$ and $\mathbf{p}$ respectively, the time-independent Schrödinger equation reads as 


$$
H\left|a>\equiv \mathbf{p}^{2}\right| a>=E_{a} \mid a>
$$

Here, $a$ stands for an arbitrary but complete set of quantum numbers. Examples are the place, the momentum and the energy-angular momentum representations, each with three quantum numbers. The vectors $|a\rangle$, being eigenvectors of a Hermitian operator, are assumed to form an orthonormal basis in the Hilbert space of square integrable functions over $\mathbf{R}^{3}$. We express this symbolically by $<a\left|a^{\prime}\right\rangle=\delta\left(a-a^{\prime}\right)$, where the $\delta$ should be read as Kronecker delta with respect to discrete quantum numbers.

In the following we always use potentials which are built up out of atom-like potentials centered around lattice positions $\mathbf{R}_{i}$. Therefore, it is convenient to introduce an alternative basis in Hilbert space by applying the unitary shift operator:

$$
\left|a, i>=e^{-i \mathbf{p} \cdot \mathbf{R}_{i}}\right| a>
$$

The functions $\mid a, i>$ are clearly centered around $\mathbf{R}_{i}$ because

$$
<\mathbf{r}|a, i\rangle=\left\langle\mathbf{r}-\mathbf{R}_{i} \mid a\right\rangle
$$

We note that for all $i$ the operator $\sum_{a}|a, i><a, i|$ equals the unity operator. In this notation the symbol $\Sigma$ is to be interpreted in the appropriate way. For example, in the energy-angular momentum representation with quantum numbers $(E, l, m) \equiv(E, L)$ we have

$$
\sum_{a} \sim \sum_{L} \int_{0}^{\infty} d E
$$

The wave functions $|a, i\rangle$ and $|a, j\rangle$ are not orthogonal for $i \neq j$. This defines a matrix $J$ by

$$
<a, i \mid a^{\prime}, j>=J_{a a^{\prime}}^{i j} \delta\left(E_{a}-E_{a^{\prime}}\right)
$$

We may explicitly write a delta function here, because the shift operator $\exp \left(-i \mathbf{p} \cdot \mathbf{R}_{i}\right)$ commutes with the free space hamiltonian.

In the following we shall frequently make use of the free space Green's operator defined by

$$
G(E) \equiv(E+i \varepsilon-H)^{-1} \equiv\left(E^{+}-H\right)^{-1}
$$

with $\varepsilon$ small and positive. Matrix elements of $G$ can readily be expressed in terms of the matrix $J$ :

$$
\left.<a, i|G(E)| a^{\prime}, j\right\rangle \equiv G_{a a^{\prime}}^{i j}(E)=\frac{1}{E^{+}-E_{a}} J_{a a^{\prime}}^{i j} \delta\left(E_{a}-E_{a^{\prime}}\right)
$$

As for the notation, we emphasize already here that the matrix $G$, depending on three energies, should 
be distinguished from a matrix $\bar{G}$, to be defined later on and which depends on only one energy.

From definition (2.5) for the $J$ matrix it directly follows that

$$
J_{a a^{\prime}}^{i j}=\sum_{a^{\prime \prime}} J_{a a^{\prime \prime}}^{i^{\prime \prime}} J_{a^{\prime} a^{\prime}}^{i^{\prime} i^{\prime}}
$$

under the condition $E_{a}=E_{a^{\prime}}=E_{a^{\prime \prime}}$. A similar property for the $G$ matrix reads as

$$
G_{a a^{\prime}}^{i j}=\sum_{a^{\prime \prime}} G_{a a^{\prime \prime}}^{i i^{\prime \prime}} J_{a^{\prime} a^{\prime}}^{i^{\prime} j}
$$

In section 3 we shall make use of these expansions. We note that in both equations no spatial conditions on the vector $\mathbf{R}_{i}$ are in force.

\subsection{One Scatterer}

To study potential-scattering we introduce a potential $V^{i}$, centered around $\mathbf{R}_{i}$. We put no other restrictions on $V^{i}$ than that it vanishes outside a finite region $S^{i}$ around $\mathbf{R}_{i}$ and behaves sufficiently smoothly in the near vicinity of $\mathbf{R}_{i}$, so that the eigenstates $\mid a, i,+>$ of the hamiltonian $H+V^{i}$ are also elements of Hilbert space. The states $\mid a, i,+>$ are assumed to form an orthonormal basis. They are connected to the free space states $|a, i\rangle$ via a Lipmann-Schwinger equation:

$$
|a, i,+>=| a, i>+G\left(E_{a}\right) V^{i} \mid a, i,+>
$$

This can be rewritten into the form

$$
\left(1-G\left(E_{a}\right) V^{i}\right)|a, i,+>=| a, i>
$$

from which it follows that the operator $\left(1-G V^{i}\right)$ is unitary. In scattering theory it is common to introduce the $t$-matrix of $V^{i}$. For example, in the KKR equations all potential dependent information is contained in the $t$-matrices of the individual scatters. The $t$-matrix $t^{i}$ corresponding to $V^{i}$ is defined by

$$
t^{i}\left|a, i>\equiv V^{i}\right| a, i,+>
$$

with matrix elements

$$
t_{a a^{\prime}}^{i}=\left\langle a, i\left|t^{i}\right| a^{\prime}, i\right\rangle
$$

Using definition (2.11a) of the $t$-matrix we may rewrite equation (2.10a) into the form 


$$
\left|a, i,+>=\left(1+G\left(E_{a}\right) t^{i}\right)\right| a, i>,
$$

from which we conclude that the operator $\left(1+G\left(E_{a}\right) t^{i}\right)$ is unitary. This property is used later on to study the determinant of the $t$-matrices.

\subsection{Perfect Crystal}

The crystal potential is given by

$$
V^{c r}=\sum_{i} V^{i}
$$

with $i$ running over lattice positions. As is common, we assume the electron-electron interactions to be included in the construction of the $V^{i}$. We put here no other restrictions on the $V^{i}$ than in the preceding subsection. In particular, we allow the $V^{i}$ to be spatially overlapping. In spite of its infinite size, the perfect crystal is mathematically tractable thanks to the translation symmetry. The crystal wave functions $\left|\psi^{c r}\right\rangle$, being eigenstates of the hamiltonian $H+V^{c r}$, can be labeled by $k \equiv(n, \mathbf{k})$ with $n$ the band index and $\mathbf{k}$ crystal momentum. The Bloch theorem states that they obey the following periodicity property

$$
\left\langle\mathbf{r}-\mathbf{R}_{i} \mid \psi_{k}^{c r}\right\rangle=\exp \left(-i \mathbf{k} \cdot\left(\mathbf{R}_{i}-\mathbf{R}_{j}\right)\right)<\mathbf{r}-\mathbf{R}_{j}\left|\psi_{k}^{c r}\right\rangle
$$

Taking free space as the reference system, $\left|\psi_{k}^{c r}\right\rangle$ satisfies the Lippman-Schwinger equation

$$
\left|\psi_{k}^{c r}\right\rangle=\sum_{i} G V^{i}\left|\psi_{k}^{c r}\right\rangle
$$

with $|\psi k\rangle$ and $G$ evaluated at the same energy . Note that, in contrast with equation (2.10a), the socalled incident wave has to be omitted here in view of the infinite dimensions of the crystal.

One of the basic concepts of the theory is the development of $\left|\psi_{k}^{c r}\right\rangle$ within $S^{i}$ in terms of the basis set $\{\mid a, i,+>\}$, introduced in section 2.2. Because $\left|\psi_{k}^{c r}\right\rangle$ is not normalizable over $\mathbf{R}^{3}$ we introduce a cut-off version $\left|\psi_{k}^{c r}\right\rangle^{i}$ of the wave function by

$$
\begin{aligned}
\left\langle\mathbf{r} \mid \psi_{k}^{c r}\right\rangle^{i} & =\left\langle\mathbf{r} \mid \psi_{k}^{c r}\right\rangle \text { if } \mathbf{r} \in S^{i} \\
& =0 \quad \text { if } \mathbf{r} \notin S^{i}
\end{aligned}
$$

Because $\left|\psi_{k}^{c}\right\rangle^{i}$ is an element of Hilbertspace, it can be written as

$$
\left|\Psi_{k}^{c r}\right\rangle^{i}=\sum_{a} \mid a, i,+>d_{a, k}^{i}
$$

with the coefficients $d$ given by 


$$
\begin{aligned}
d_{a, k}^{i} & =\left\langle a, i,+\mid \psi_{k}^{c r}\right\rangle^{i} \\
& =\int d \mathbf{r}<a, i,+|\mathbf{r}\rangle\left\langle\mathbf{r} \mid \psi_{k}^{c r}\right\rangle^{i}
\end{aligned}
$$

It is important to realize that in this expansion basis functions at all energies are involved. The only way to avoid this complication is to require the $S^{i}$ not to overlap. The consequences of that assumption are studied in section 3 . Let us write equation (2.15) into the form

$$
\left(1-G V^{j}\right)\left|\psi_{k}^{c r}\right\rangle=\sum_{i \neq j} G V^{i}\left|\psi_{k}^{c r}\right\rangle
$$

It is appropriate to apply here an idea introduced by Brown and Ciftan (1983), who multiplied both sides of equation $(2.17 \mathrm{a})$ by the same factor :

$$
\left.\left.<a, j,+\left|V^{j}\left(1-G V^{j}\right)\right| \psi_{k}^{c r}\right\rangle=\sum_{i \neq j}<a, j,+\left|V^{i} G V^{i}\right| \psi_{k}^{c r}\right\rangle
$$

We shall deal with the left and right hand sides of this equation separately. Inserting an unity operator in the place representation into the left hand side of equation $(2.17 \mathrm{~b})$ we obtain

$$
\int d \mathbf{r}<a, j,+\left|V^{j}\left(1-G V^{j}\right)\right| \mathbf{r}><\mathbf{r}\left|\psi_{k}^{c r}\right\rangle
$$

In view of the finite range of $V^{j}$ the $\mathbf{r}$ integration is restricted to $S^{j}$, so we may replace $\left|\psi k_{k}^{r}\right\rangle$ by $\left|\psi \psi_{k}\right\rangle^{j}$ in this expression. Inserting an unity operator yields

$$
\int d \mathbf{r} \sum_{a^{\prime}}<\boldsymbol{a}, j,+\left|V^{j}\left(1-G V^{j}\right)\right| a^{\prime}, j,+><a^{\prime}, j,+|\mathbf{r}><\mathbf{r}| \psi_{k}^{c r}>^{j}
$$

Note that integration and summation may be interchanged here, because both correspond to expansions in terms of orthonormal bases in Hilbertspace. For the right hand side of equation (2.174a similar procedure can be followed, which leads to the expression :

$$
\left.\left.\sum_{i \neq j} \int d \mathbf{r} \sum_{a^{\prime}, a^{\prime \prime}, a^{\prime \prime \prime}}<a, j,+\left|V^{j}\right| a^{\prime}, j\right\rangle<a^{\prime}, j|G| a^{\prime \prime}, i><a^{\prime \prime}, i\left|V^{i}\right| a^{\prime \prime \prime}, i,+><a^{\prime \prime \prime}, i,+|\mathbf{r}><\mathbf{r}| \psi_{k}^{c r}\right\rangle^{i}
$$

In view of equations $(2.7),(2.10 \mathrm{~b}),(2.11)$, and (2.16c) we may reduce expressions (2.18b) and (2.19) considerably. After this reduction we arrive at an equation, which plays a central rôle in the theory:

$$
\sum_{a^{\prime}}\left(t^{\dagger}\right) d a^{\prime} d \dot{a}^{\prime}, k=\sum_{i \neq j} \sum_{a^{\prime}, a^{\prime \prime}, a^{\prime \prime \prime}}\left(t^{\dagger}\right) \dot{d a^{\prime}} G^{i a^{\prime \prime}} a^{\prime \prime} t_{a^{\prime \prime} a^{\prime \prime \prime}}^{i^{\prime}} d_{a^{\prime \prime \prime}, k}^{i}
$$

If we omit for the moment the index $k$ in the coefficients $d$, equation (2.20) is valid for an arbitrary set of scatterers, if distributed over the whole space. 
In matrix notation equation $(2.20)$ can be written as

$$
M d=0
$$

with $M$ given by

$$
M_{a, a^{\prime}}^{i j}=\left(t^{\dagger}-t^{\dagger} G t\right)_{a, a^{\prime}}^{i j}
$$

In equation (2.21) the summations run both over sites and quantum numbers. For the $t$-matrix we used the convention

$$
t^{i j}=t^{i} \delta_{i, j}
$$

and for $G$ we introduced the restriction

$$
G^{i j}=0 \text { if } i=j
$$

From equation (2.21) we deduce that a non-trivial solution for the coefficients only exists if

$$
\operatorname{det}_{i, a}(M)=0
$$

where the indices denote that the determinant is taken both with respect to sites and quantum numbers.

In the perfect crystal the sites $R_{i}$ are restricted to lattice positions and the Bloch condition (2.14) holds. Then we meet with the Fourier transform $\mathcal{G}$ of $G$ given by

$$
\mathcal{S}_{a, a^{\prime}}(E, \mathbf{k})=\sum_{i \neq j} G_{a a^{\prime}}^{i j}(E) \exp \left(i \mathbf{k} \cdot\left(\mathbf{R}_{i}-\mathbf{R}_{j}\right)\right)
$$

In the crystal all $t$-matrices are equal, so we may drop the site index of $t$. Then, equation (2.21) reads

$$
\mathscr{M}(E, \mathbf{k}) d_{k}=0
$$

with $\mathscr{K}$ given by

$$
\mathscr{M}_{a, a^{\prime}}(E, \mathbf{k})=\left(t^{\dagger}-t^{\dagger} \mathcal{S}(E, \mathbf{k}) t\right)_{a, a^{\prime}}
$$

Condition (2.25) reduces to

$$
\operatorname{det}_{a}(\mathfrak{K})=0
$$

with the determinant taken with respect to quantum numbers only. From equation (2.28) we see that this condition is satisfied either if 


$$
\operatorname{det}_{a}\left(t^{\dagger}\right)=0
$$

or if

$$
\operatorname{det}_{a}(1-\mathcal{G}(E, \mathbf{k}) t)=0
$$

Condition (2.30a) is trivial, because it holds for all potentials $\mathrm{V}$, which tend to zero at infinity. This immediately follows from the relation

$$
\left.<a|t| a^{\prime}\right\rangle=<a|V| a^{\prime},+>=\sum_{a^{\prime \prime}}<a|V| a^{\prime \prime}><a^{\prime \prime}\left|\left(1+G\left(E_{a^{\prime}}\right) t\right)\right| a^{\prime}>
$$

As shown above, the operator $(1+G t)$ is unitary, so we have the relation:

$$
\operatorname{det}(t)=\operatorname{det}(V)
$$

In the place representation the elements of the operator $V$ are given by

$$
<\mathbf{r}|V| \mathbf{r}^{\prime}>=V(\mathbf{r}) \delta\left(\mathbf{r}-\mathbf{r}^{\prime}\right)
$$

from which we find that $\operatorname{det}(V)=0$ if $V(\mathbf{r}) \rightarrow 0$ for $\mathbf{r} \rightarrow \infty$.

The presence of condition (2.30a) as a possible solution of equation (2.29) deserves further analysis. In the context of this paper we are only interested in condition $(2.30 \mathrm{~b})$. It strongly resembles the well-known KKR equations, though it is a generalised version, because now also the off-the-energyshell components of the band structure matrix are involved.

\section{\$3. Multiple Scattering Theory for Non-Overlapping Potentials}

In this section we introduce the assumption of non-overlapping potentials and specify the representation to be the energy-angular momentum representation with quantum numbers $(E, l, m) \equiv(E, L)$. Then it appears to be possible to bring the generalised KKR equation (2.29) into the well-known on-theenergy-shell form.

\subsection{Non-overlapping potentials.}

If the ranges $S^{i}$ of the potentials $V^{i}$ have no spatial overlap, the crystal potential $\sum_{j} V^{j}$ coincides within $S^{i}$ with $V^{i}$. This allows an expansion of the crystal wave function $\left|\psi_{k}^{c r}\right\rangle$ in terms of only those basis functions $\mid a, i,+>$ with $E_{a}=E$ with $E$ the energy under consideration. So, within $S^{i}$ we may use the following on-the-energy-shell expansion: 


$$
\left|\psi_{k}^{c r}>=\sum_{a}\right| a, i,+>c_{a, k}^{i} \delta\left(E_{a}-E\right)
$$

It is the aim of "classical" KKR theory to calculate the coefficients $c^{i}$. We note that in the general theory, presented in section 2 , the assumption of non-overlapping potentials simply implies the replacement

$$
d_{a, k}^{i} \rightarrow c_{a, k}^{i} \delta\left(E_{a}-E\right)
$$

\subsection{The $(E, L)$ representation}

In the theory developed above the free space basis functions $|a, i\rangle$ play an important rôle. In the energy-angular momentum representation with $a \equiv(E, L)$ these functions are given by

$$
<\mathbf{r}+\mathbf{R}_{i} \mid E, L, i>=\frac{E^{1 / 4}}{\pi^{1 / 2}} j_{l}(\sqrt{E}|\mathbf{r}|) Y_{L}(\hat{r}) \equiv \frac{E^{1 / 4}}{\pi^{1 / 2}} j_{L}(E, \mathbf{r})
$$

The $j_{l}$ and $Y_{L}$ stand for the spherical Bessel function and real spherical harmonic respectively. With these conventions it holds that $\left\langle E, L, i \mid E^{\prime}, L^{\prime}, i\right\rangle=\delta\left(E-E^{\prime}\right) \delta_{L, L^{\prime}}$. For the matrix $J$, defined in equation (2.5), it is derived in appendix A that

$$
J_{L L^{\prime}}\left(E, \mathbf{R}_{i j}\right) \equiv J_{E L, E L^{\prime}}^{i j}=4 \pi \sum_{L^{\prime \prime}} i^{\left(l-l^{\prime \prime}+l^{\prime \prime}\right)} c_{L L^{\prime} L^{\prime \prime}} j_{L^{\prime \prime}}\left(E, \mathbf{R}_{i j}\right)
$$

with $\mathbf{R}_{i j}=\mathbf{R}_{i}-\mathbf{R}_{j}$.

The $c_{L L^{\prime} L^{\prime \prime}}$ are Gaunt coefficients which are non-vanishing only if $l+l^{\prime}+l^{\prime \prime}$ is even and $\left|l-l^{\prime}\right| \leqslant l^{\prime \prime} \leqslant\left|l+l^{\prime}\right|$. For later purpose we introduce, analogous to expression (3.4), a matrix $\bar{G}$ by

$$
\bar{G}_{L L^{\prime}}\left(E, \mathbf{R}_{i j}\right) \equiv \bar{G}_{E L, E L^{\prime}}^{i j}=4 \pi \sum_{L^{\prime \prime}} i^{\left(l-l^{\prime}+l^{\prime \prime}\right)} c_{L L^{\prime} L^{\prime \prime}} h_{L^{\prime \prime}}\left(E, \mathbf{R}_{i j}\right) \text { if } i \neq j
$$

For $i=j$ we define $\bar{G}$ to be vanishing. The Hankel function $h_{L}$ is given by $h_{L}=j_{L}+i n_{L}$ with $n_{l}$ the Neumann function. From this, we see that for $i \neq j$ the matrix $J$ equals the real part of $\bar{G}$.

The analogue of expansion (2.8) is directly found to be

$$
J_{L L^{\prime}}\left(E, \mathbf{R}_{i j}\right)=\sum_{L^{\prime \prime}} J_{L L^{\prime \prime}}\left(E, \mathbf{R}_{i i^{\prime \prime}}\right) J_{L^{\prime \prime} L^{\prime}}\left(E, \mathbf{R}_{i^{\prime} j}\right)
$$

By taking $L \equiv 0$ in expression (3.4) we find

$$
\sqrt{(4 \pi)} j_{L}(E, \mathbf{r})=J_{0 L}(E, \mathbf{r})
$$

From this a special case of expansion (3.6) is obtained: 


$$
j_{L}(E, \mathbf{r})=\sum_{L^{\prime}} j_{L^{\prime}}\left(E, \mathbf{r}^{\prime}\right) J_{L^{\prime} L}\left(E, \mathbf{r}-\mathbf{r}^{\prime}\right)
$$

The analogue of equation (2.9) can be shown to be (Molenaar,1983)

$$
\bar{G}_{L L^{\prime}}\left(E, \mathbf{R}_{i j}\right)=\sum_{L^{\prime \prime}} \bar{G}_{L L^{\prime \prime}}\left(E, \mathbf{R}_{i i^{\prime}}\right) J_{L^{\prime \prime} L^{\prime}}\left(E, \mathbf{R}_{i^{\prime} j}\right)
$$

under the condition $\quad:\left|\mathbf{R}_{i i^{\prime}}\right|>\left|\mathbf{R}_{i^{\prime} j}\right|$.

The matrix $t^{i}$, introduced in equation (2.11), is in the $(E, L)$ representation given by

$$
t_{E L, E^{\prime} L^{\prime}}^{i}=\int d \mathbf{r} \frac{E^{1 / 4}}{\pi^{1 / 2}} j_{L}(E, \mathbf{r}) V^{i}(\mathbf{r})<\mathbf{r} \mid E^{\prime}, L^{\prime},+>
$$

where we have chosen $\mathbf{R}_{i}$ to be the origin. To obtain $t^{i}$ it is necessary to calculate $\left\langle\mathbf{r} \mid E^{\prime}, L^{\prime},+\right\rangle$ by solving the Schrödinger equation numerically. Nowadays, this may be considered to be a standard technique.

Let us now study the matrix $\Re$, defined by equation $(2.28)$, in the $(E, L)$ representation. To avoid confusion in the notation we shall denote the energy in the argument of this matrix by $\mathrm{E}_{0} \quad$ Writing all indices explicitly, $\mathscr{T}$ is given by

$$
\mathfrak{M}_{E L, E^{\prime} L^{\prime}}\left(E_{0}, \mathbf{k}\right)=t_{E L, E^{\prime} L^{\prime}}^{t}-\sum_{L^{\prime \prime}, L^{\prime \prime}} \int_{0}^{\infty} d E^{\prime \prime} t_{E L, E^{\prime \prime} L^{\prime \prime}} \mathcal{S}_{E^{\prime \prime} L^{\prime \prime}, E^{\prime \prime} L^{\prime \prime \prime}}\left(E_{0}, \mathbf{k}\right) t_{E^{\prime \prime} L^{\prime \prime}, E^{\prime} L^{\prime}}
$$

with the matrix $\mathfrak{S}$, according to equation (2.26), defined by

$$
\begin{aligned}
\mathcal{S}_{E L, E L^{\prime}}\left(E_{0}, \mathbf{k}\right) & =\sum_{i \neq j} G_{E L, E L^{\prime}}\left(E_{0}, \mathbf{R}_{i j}\right) \exp \left(i \mathbf{k} \cdot \mathbf{R}_{i j}\right) \\
& =\sum_{i \neq j} \frac{J_{E L, E L^{\prime}}\left(E, \mathbf{R}_{i j}\right)}{\left(E_{0}^{+}-E\right)} \exp \left(i \mathbf{k} \cdot \mathbf{R}_{i j}\right) .
\end{aligned}
$$

The latter equality immediately follows from relation (2.7). The determinant in equation (2.29) is taken with respect to both $E$ and $L$ indices of the matrix $\mathfrak{N}$. However, in case of non-overlapping potentials we may insert substitution (3.2) into equation (2.20). It is straightforward to check that in that case the determinant in condition (2.29) has to be taken with respect to angular momentum indices only and expression (3.11) is to be used with $E=E^{\prime}=E_{0}$. We indicate this reduction as follows:

$$
\operatorname{det}_{L}\left[\Re\left(E_{0}, \mathbf{k}\right)\right]=0
$$

This expression strongly resembles the well-known KKR condition, except for the energy integration present in the r.h.s. of equation (3.11). If we isolate those components in the product $t^{\dagger} \mathcal{G} t$, which are 
involved in this integration, we have to evaluate the following integrals:

$$
\int_{0}^{\infty} d E j_{L}\left(E, \mathbf{r}-\mathbf{R}_{i}\right) \frac{J_{L L^{\prime}}\left(E, \mathbf{R}_{i j}\right)}{\left(E_{0}^{+}-E\right)} j_{L^{\prime}}\left(E, \mathbf{r}^{\prime}-\mathbf{R}_{j}\right)
$$

with $\mathbf{r}$ and $\mathbf{r}^{\prime}$ running over $S^{i}$ and $S^{j}$ respectively $(i \neq j)$. In view of expression (3.4) for the $J$ matrix and definition (3.3) of $j_{L}$, the integrand contains the product of three spherical Bessel functions with arguments $\sqrt{E}\left|\mathbf{r}-\mathbf{R}_{i}\right|, \sqrt{E}\left|\mathbf{R}_{i j}\right|$ and $\sqrt{E}\left|\mathbf{r}^{\prime}-\mathbf{R}_{j}\right|$. If $i$ and $j$ refer to non-neighbouring sites we have that $\left|\mathbf{r}-\mathbf{R}_{i}\right|+\left|\mathbf{r}^{\prime}-\mathbf{R}_{j}\right|<\left|\mathbf{R}_{i j}\right|$. Then, all conditions of the theorem in appendix $\mathrm{B}$ are satisfied, thanks to the properties of the Gaunt coefficients. Application of this theorem transforms expression (3.14) into the expression

$$
j_{L}\left(E_{0}, \mathbf{r}-\mathbf{R}_{i}\right) \bar{G}_{L L^{\prime}}\left(E_{0}, \mathbf{R}_{i j}\right) j_{L^{\prime}}\left(E_{0}, \mathbf{r}^{\prime}-\mathbf{R}_{j}\right)
$$

with $\bar{G}$ defined by equation (3.5).

If $\mathbf{R}_{i}$ and $\mathbf{R}_{j}$ are neighbouring sites the theorem of appendix $\mathrm{B}$ is not directly applicable, because the spatial condition is not fulfilled. To remedy this we follow an idea by Gonis (1986) and introduce "farfield" centres $\mathbf{R}_{i^{\prime}}$ and $\mathbf{R}_{j^{\prime}}$ by

$$
\begin{aligned}
& \mathbf{R}_{i^{\prime}}=1 / 2\left(\mathbf{R}_{i}+\mathbf{R}_{j}\right)+A\left(\mathbf{R}_{i}-\mathbf{R}_{j}\right) \\
& \mathbf{R}_{j^{\prime}}=1 / 2\left(\mathbf{R}_{i}+\mathbf{R}_{j}\right)-A\left(\mathbf{R}_{i}-\mathbf{R}_{j}\right)
\end{aligned}
$$

where $A$ is an arbitrarily big, real number. Thanks to properties (3.6) and (3.8) we may in the integrand of (3.14) replace $\mathbf{R}_{i}$ and $\mathbf{R}_{j}$ by $\mathbf{R}_{i^{\prime}}$ and $\mathbf{R}_{i^{\prime}}$ respectively. It is interesting to notice that the same replacement could have been made in equation (2.19). In the latter context it is the more obvious that this is allowed, because there it corresponds to replacing some unity operators by other ones. After the replacements we have $\left|\mathbf{r}-\mathbf{R}_{i^{\prime}}\right|+\left|\mathbf{r}^{\prime}-\mathbf{R}_{j^{\prime}}\right|<\left|\mathbf{R}_{i^{\prime} j^{\prime}}\right|$ for nearly all points $\mathbf{r}$ and $\mathbf{r}^{\prime}$. Excluding for the moment the regions of $S_{i}$ and $S_{j}$ where this condition is violated yet, we may again apply the theorem of appendix B.

As before, this results in an expression like (3.15) with $\mathbf{R}_{i^{\prime}}$ and $\mathbf{R}_{j^{\prime}}$ instead of $\mathbf{R}_{i}$ and $\mathbf{R}_{j}$. Using expansion (3.8) we may rewrite this into the form

$$
j_{L}\left(\mathbf{r}-\mathbf{R}_{i}\right) J_{L L^{\prime}}\left(\mathbf{R}_{i i^{\prime}}\right) \bar{G}_{L^{\prime} L^{\prime \prime}}\left(\mathbf{R}_{i^{\prime} j^{\prime}}\right) J_{L^{\prime \prime} L^{\prime \prime \prime}}\left(\mathbf{R}_{i^{\prime} j}\right) j_{L^{\prime \prime \prime}}\left(\mathbf{r}^{\prime}-\mathbf{R}_{j}\right)
$$

In expression (3.17) all functions are to be evaluated at energy $E_{0}$. One should be careful in performing the summations over the angular momenta in this expression, since otherwise convergence is not assured. 
Substituting expressions (3.15) and (3.17) into equation (3.11), we obtain an on-the-energy-shell expression for $\Re$ :

$$
\mathscr{T}_{L L^{\prime}}\left(E_{0}, \mathbf{k}\right)=t_{L L^{\prime}}^{t}-\sum_{L^{\prime \prime}, L^{\prime \prime \prime}} t_{L L^{\prime \prime}}^{\dagger} \overline{\mathcal{S}}_{L^{\prime \prime} L^{\prime \prime \prime}}\left(E_{0}, \mathbf{k}\right) t_{L^{\prime \prime} L^{\prime}}
$$

The matrix $\overline{\mathfrak{S}}$ is given by

$$
\begin{aligned}
\overline{\mathfrak{S}}_{L L^{\prime}}\left(E_{0}, \mathbf{k}\right)= & \sum_{i \in I_{1}} \bar{G}_{L L^{\prime}}\left(E_{0}, \mathbf{R}_{i j}\right) \exp \left(i \mathbf{k} \cdot \mathbf{R}_{i j}\right) \\
& +\sum_{i \in I_{2}} \sum_{L^{\prime \prime}, L^{\prime \prime \prime}} J_{L L^{\prime \prime}}\left(E_{0}, \mathbf{R}_{i i^{\prime}}\right) \bar{G}_{L^{\prime \prime}, L^{\prime \prime}}\left(E_{0}, \mathbf{R}_{i^{\prime} j^{\prime}}\right) J_{L^{\prime \prime \prime} L^{\prime}}\left(E_{0}, \mathbf{R}_{j^{\prime} j}\right) \exp \left(i \mathbf{k} \cdot \mathbf{R}_{i j}\right)
\end{aligned}
$$

Here, we denote by $I_{2}$ the lattice positions neighbouring to $\mathbf{R}_{j}$ and by $I_{1}$ all other positions apart from $\mathbf{R}_{j}$ itself.

As in the general case (2.29), condition (3.13) has the trivial solution $\operatorname{det}_{L}\left(t^{\dagger}\right)=0$. This condition does clearly not contain any band structure information. A second solution reads as

$$
\operatorname{det}_{L}\left(1-\overline{\mathfrak{S}}\left(E_{0}, \mathbf{k}\right) t\right)=0
$$

Here, we meet with the product $\overline{\mathfrak{g}} t$. In this product the angular momentum summations may be performed in any order by use of contraction (3.9). This may be checked by observing that the spatial restrictions are never violated. So the matrix $\overline{\mathscr{G}}$ reduces in the context of condition (3.20) to the well-known KKR band structure matrix, given by

$$
\overline{\mathfrak{S}}_{L L^{\prime}}\left(E_{0}, \mathbf{k}\right)=\sum_{i \neq j} \bar{G}_{L L^{\prime}}\left(E_{0}, \mathbf{R}_{i j}\right) \exp \left(i \mathbf{k} \cdot \mathbf{R}_{i j}\right)
$$

Because the considerations in formulae (3.16)-(3.21) are independent of the value of $A$ in equation (3.16), we may take the limit $A \rightarrow \infty$, thus showing that KKR condition (3.20) with (3.21) holds, also if the ranges $S^{i}$ and $S^{j}$ coincide with the $i$-th and $j$-th Wigner-Seitz cells respectively.

In shorthand notation condition (3.20) could be written as $\operatorname{det}_{L}(\overrightarrow{\mathrm{S}} t)=1$. It is interesting to realize that, if the angular momentum indices extend to infinity, one has $\operatorname{det}_{L}(t) \rightarrow 0$ and $\operatorname{det}_{L}(\overline{\mathcal{S}}) \rightarrow \infty$, but apparently $\operatorname{det}_{L}(\overline{\mathcal{G}} t) \rightarrow 1$.

To avoid conceptual problems one could introduce an arbitrarily large but finite cut-off value for angular momentum but this does not change the essential features of the present derivations. 


\section{\$4. Discussion}

The discussion on Near-Field corrections until now has made clear that problems in going beyond the Muffin-Tin approximation stem from the spatial conditions in the following matrix elements of the free space Green's function:

$$
\begin{aligned}
<\mathbf{r}|G(E)| \mathbf{r}^{\prime}> & =-i \sqrt{E} \sum_{L} j_{L}(E, \mathbf{r}) h_{L}\left(E, \mathbf{r}^{\prime}\right) \text { if } r<r^{\prime} \\
& =-i \sqrt{E} \sum_{L} j_{L}\left(E, \mathbf{r}^{\prime}\right) h_{L}(E, \mathbf{r}) \text { if } r^{\prime}<r .
\end{aligned}
$$

These expansions are at one energy and lead to spatial conditions in similar expansions such as

$$
\left.<\mathbf{r}|G(E)| \mathbf{r}^{\prime}\right\rangle=\sum_{L, L^{\prime}} j_{L}\left(E, \mathbf{r}-\mathbf{R}_{i}\right) \bar{G}_{L L^{\prime}}\left(E, \mathbf{R}_{i j}\right) j_{L^{\prime}}\left(E, \mathbf{r}^{\prime}-\mathbf{R}_{j}\right)
$$

in which, for neighbouring sites $i$ and $j$, the summations are unconditionally convergent only if $\mathbf{r}$ and $\mathbf{r}^{\prime}$ are restricted to Muffin-Tin spheres around $R_{i}$ and $R_{j}$ respectively. However, it is shown above that these restrictions need not to be obstructive to derive the KKR equation for non-Muffin-Tin potentials.

In this paper a short discussion of preceding work on this subject may not be missing. Williams and van Morgan (1972) used an expansion such as (4.2) outside the region where it holds. On detecting this error, Ziesche (1974) and Faulkner (1979) concluded to the existence of Near-Field corrections if one goes beyond the Muffin-Tin approximation. Impressive calculations by Faulkner $(1985,1986)$ suggested that the corrections, if any, are small. Neglecting possible corrections he performed an empty lattice analysis in two dimensions. Although he made use of an cut-off value as big as $l_{\max }=60$ this numerical approach did not exclude the existence of the corrections in a definite way.

Brown and Ciftan $(1983,1985,1986)$ modified the theoretical basis of the theory a bit. They used in expansion (3.1) basis functions, which are connected to the part of the crystal potential within the circumscribing spheres of the Wigner-Seitz cells. They carefully applied these expansions only within the cells. In this way KKR-like equations can be obtained, which will certainly yield the correct band structure. However, the wave function coefficients and the $t$-matrices in this approach are quite different from the corresponding quantities in KKR theory.

A completely different approach is followed by Gonis (1986). The essence of his method is also used in the present paper. In expansions around neighbouring sites he moves their centres to far field positions at opposite sides of and at a far distance from the original positions, just as indicated in formula (3.16). In this way one gets rid of the spatial conditions in expansion (4.2). His derivation concerns a system consisting of two non-overlapping potentials in free space, and it is claimed that it can be extended to the crystal. Although the setting of the present paper is quite different, it in fact includes this extension. Here, we avoid a complication present in the original two-scatterer approach, where use is made of the inverse of the $t$-matrices of the individual scatterers. However, for potentials of finite range 
one has $\operatorname{det}_{L}(t)=0$ if the $L, L^{\prime}$ indices in $t_{L L^{\prime}}$ extend to infinity. The only way to remedy this problem is to introduce an artificial cut-off value for angular momentum indices. But then it is not allowed to shift the far field positions to infinity and it is formally not proved that the Near Field corrections vanish.

A recent contribution is by Zeller (1987). He introduces in expansion (4.2) a factor $f(N)$, which assures absolute convergence as long as $N$ is finite. Then, the usual KKR equation can be derived including $f(N)$. After all, one takes $N$ very large upon which $f(N)$ approaches the value one. Zeller presents a thorough analysis of the convergence properties of the several angular momentum summations in the theory. From this insight he discusses that the Near Field corrections can be ignored from a practical point of view but are formally non-vanishing.

After a discussion of about one and a half decade we may conclude that the pendulum has swung back. After successive periods of ignoring, introducing and discussing the Near Field corrections, we now enter the stage in which the beauty and power of the KKR-formalism may be acknowledged to its full extent. The present derivation for overlapping potentials is a nice example of the phenomenon that some theories, originally developed in a restricted context, appear to have a wider range. It would be a nice subject for further research to study whether the generalized KKR equation (2.30b) can be brought into a tractable form.

\section{Acknowledgement}

I would like to thank A. Lodder from the Free University, Amsterdam, for drawing my attention to the dicussion on Near-Field corrections, helpful comments and critical reading of the manuscript. 


\section{References}

1) R.G. Brown and M. Ciftan, Phys. Rev. B. 27, 4564 (1983).

2) R.G. Brown and M. Ciftan, Phys. Rev. B. 32, 1343 (1985).

3) R.G. Brown and M. Ciftan, Phys. Rev. B. 33, 7937 (1986).

4) J.S. Faulkner, Phys. Rev. B. 19, 6186 (1979).

5) J.S. Faulkner, Phys. Rev. B. 32, 1339 (1985).

6) J.S. Faulkner, Phys. Rev. B. 34, 5931 (1986).

7) A. Gonis, Phys. Rev. B. 33, 5914 (1986).

8) W. Kohn and N. Rostoker, Phys. Rev. 94, 1111 (1954).

9) J. Korringa, Physica 13, 392 (1947).

10) J. Molenaar, 'Bloch Electron Scattering in Dilute $\mathrm{K}$ and $\mathrm{Cu}$ Alloys', thesis, Free University, Amsterdam (1983), available on request.

11) A.R. Williams and J.W. van Morgan, J. Phys. C. 5, L293 (1972).

12) A.R. Williams and J.W. van Morgan, J. Phys. C. I, 37 (1974)

13) R. Zeller, Institut für Festkörperforschung, Jülich, Postfach 1913, D-5170 Jülich, FDR, submitted to J. Phys. C, (1987).

14) P. Ziesche, J. Phys. C. I, 1085 (1974). 


\section{Appendix A. THE MATRIX $J$ IN THE $(E, L)$ REPRESENTATION}

In the $(E, L)$ representation the matrix $J$ with respect to the sites $\mathbf{R}_{i}$ and $\mathbf{R}_{j}$ is given by

$$
\begin{aligned}
\delta\left(E-E^{\prime}\right) J_{E L, E^{\prime} L^{\prime}}^{i j} & =\left\langle E, L, i \mid E^{\prime}, L^{\prime}, j\right\rangle \\
& =\left\langle E, L\left|\exp \left(i \mathbf{p} \cdot \mathbf{R}_{i j}\right)\right| E^{\prime}, L^{\prime}\right\rangle .
\end{aligned}
$$

For the functions $\mid E, L>$ we use the conventions embodied in eq. (3.3), thus

$$
<\mathbf{r} \mid E, L>=\frac{E^{1 / 4}}{\pi^{1 / 2}} j_{l}(\sqrt{E} r) Y_{L}(\hat{r})
$$

with the notation $r=|\mathbf{r}|$. The last member of eq. (a.1) is most easily evaluated by inserting a unity operator in the following way

$$
\int d \mathbf{k}<E, L\left|\mathbf{k}>\exp \left(i \mathbf{k} \cdot \mathbf{R}_{i j}\right)<\mathbf{k}\right| E^{\prime}, L^{\prime}>
$$

where we denote by $\mid \mathbf{k}>$ a plane wave, normalized according to $\langle\mathbf{k}| \mathbf{k}>=\delta\left(\mathbf{k}-\mathbf{k}^{\prime}\right)$, which implies

$$
<\mathbf{r} \mid \mathbf{k}>=(2 \pi)^{-3 / 2} \exp (i \mathbf{k} \cdot \mathbf{r})
$$

To evaluate the integral expression (a.3) we need the series expansion of a plane wave in terms of spherical Bessel functions and harmonics

$$
\exp (i \mathbf{k} \cdot \mathbf{r})=4 \pi \sum_{L} i^{i} j_{l}(k r) Y_{L}(\hat{k}) Y_{L}(\hat{r})
$$

To obtain the matrix element $\langle E, L| \mathbf{k}>$ we expand $<\mathbf{r} \mid \mathbf{k}>$ as follows:

$$
\begin{aligned}
<\mathbf{r}|\mathbf{k}\rangle & =\int d E \sum_{L}<\mathbf{r}|E, L><E, L| \mathbf{k}> \\
& =\int d E \sum_{L} \frac{E^{1 / 4}}{\pi^{1 / 2}} j_{l}(\sqrt{E r}) Y_{L}(\hat{r})<E, L \mid \mathbf{k}>.
\end{aligned}
$$

Comparing this expression for $\langle\mathbf{r}| \mathbf{k}>$ with the expansion found by substituting eq. (a.5) into (a.4) we conclude

$$
<E, L \mid \mathbf{k})=\frac{2^{1 / 2}}{E^{1 / 4}} i^{l} Y_{L}(\hat{k}) \delta\left(E-k^{2}\right)
$$

By means of equations (a.5) and (a.7) expression (a.3) for the $J$ matrix can be evaluated, giving 


$$
J_{E L, E^{\prime} L^{\prime}}^{i j}=4 \pi \sum_{L^{\prime \prime}} i^{\left(l-l^{\prime}+l^{\prime \prime}\right)} c_{L L^{\prime} L^{\prime \prime}} j_{L^{\prime \prime}}\left(E, \mathbf{R}_{i j}\right)
$$

with the Gaunt coefficients $c_{L L^{\prime} L^{\prime \prime}}$ defined by

$$
c_{L L^{\prime} L^{\prime \prime}}=\int d \hat{k} Y_{L}(\hat{k}) Y_{L^{\prime}}(\hat{k}) Y_{L^{\prime \prime}}(\hat{k})
$$




\section{Appendix B.}

In this appendix we prove the following proposition.

Let $I$ be defined by

$$
I=\int_{0}^{\infty} d k f(k)\left(E+i \varepsilon-k^{2}\right)^{-1} \prod_{i=1}^{n} j_{l_{i}}\left(k r_{i}\right)
$$

with $j_{l_{l}}$ spherical Bessel functions, $E$ a constant and $\varepsilon$ infinitesimally positive.

Under the following restrictions

$$
\sum_{i=1}^{n} l_{i} \text { is even }
$$

$f(k)$ is an even function of $k$

$$
\sum_{i=1}^{n-1} r_{i}<r_{n} \neq 0
$$

$$
\sum_{i=1}^{n-1} l_{i}+p \geqslant l_{n}+1
$$

$$
n+2>t
$$

where $p$ and $t$ are defined by the asymptotic behaviour of $f$ i.e.

$$
f(z) \underset{z \rightarrow 0}{\rightarrow} z^{p} \text { and } f(z) \underset{z \rightarrow \infty}{\rightarrow} z^{t}
$$

it holds, in the limit $\varepsilon \downarrow 0$, that

$$
I=-1 / 2 \pi i \frac{f(\sqrt{E})}{\sqrt{E}} h_{l_{n}}\left(\sqrt{E} r_{n}\right) \pi_{i=1}^{n-1} j_{l_{i}}\left(\sqrt{E} r_{i}\right)
$$

with $h_{l}=j_{l}+i n_{l}$ a spherical Hankel function and $n_{l}$ a spherical Neumann function. 
Proof.

We need the following properties of $j_{l}, n_{l}$ and $h_{l}$.

$$
\begin{aligned}
& j_{l}(z) \underset{z \rightarrow \infty}{\rightarrow} \frac{\cos (z-1 / 2(l+1) \pi)}{z} ; j_{l}(z) \underset{z \rightarrow 0}{\rightarrow} \frac{z^{l}}{(2 l+1) ! !} \\
& h_{l}(z) \underset{z \rightarrow \infty}{\rightarrow} \frac{\exp (i(z-1 / 2(l+1) \pi)}{z} ; h_{l} \underset{z \rightarrow 0}{\rightarrow} \frac{(2 l-1) ! !}{z^{l+1}} \\
& j_{l}(-x)=(-1)^{l} j_{l}(x) \\
& n_{l}(-x)=(-1)^{l+1} n_{l}(x) .
\end{aligned}
$$

On account of restrictions (b.2) and (b.3) and property (b.11) we may extend the integration interval to the range $-\infty<k<+\infty$. In view of property (b.12) of the spherical Neumann function we may at the same time replace one of the $j_{l}$ by $h_{l}$, for which we choose the $j_{l}(k r)$ with the largest srgument, i.e. $j_{l}\left(k r_{n}\right)$. The $h_{l_{n}}$, introduced in this way, diverges at the origin as $\left(k r_{n}\right)^{-\left(l_{n}+1\right)}$ (see (b.10)) but the product of the remaining spherical Bessel functions, the function $f(k)$ and $h_{l_{n}}$ does not show this divergent behaviour in view of restriction (b.5) with the properties (b.7) and (b.9).

Let us not close the contour by means of a semicircle in the upperhalf plane. The asymptotic behaviour for $k \rightarrow \infty$ of $j_{l}$ and $h_{l}$, given by (b.9) and (b.10), guarantees that, in view of restrictions (b.4) and (b.6), the contribution of the arc vanishes if its radius goes to infinity. The contour encloses the pole at $k_{0}=\sqrt{E}(1+i \varepsilon / E)^{1 / 2}$ which in the limit $\varepsilon \downarrow 0$ becomes $k_{0}=\sqrt{E}$. The residue of $\left(E+i \varepsilon-k^{2}\right)^{-1}$ at this pole is $(2 \sqrt{E})^{-1}$. Application of the integral theorem of Cauchy completes the proof. 